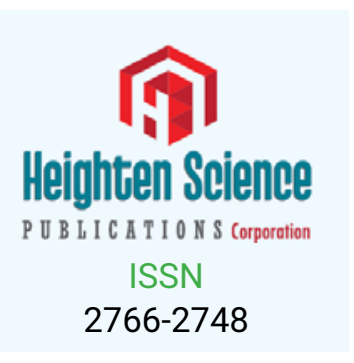

*Address for Correspondence: Boris $S$ Dizhechko, Department of Physics, MV Lomonosov, Moscow State University, City of Sterlitamak, Bashkortostan, Russia, Tel: +7(495)939-16-82; Email: fizika3000@yandex.ru

Submitted: 18 February 2019

Approved: 27 March 2019

Published: 28 March 2019

Copyright: $\odot 2019$ Dizhechko BS. This is an open access article distributed under the Creative Commons Attribution License, which permits unrestricted use, distribution, and reproduction in any medium, provided the original work is properly cited

(T) Check for updates
Opinion

\section{Moving space-matter as the basis of the intelligence in the Physical World}

\author{
Boris S Dizhechko* \\ Department of Physics, MV Lomonosov, Moscow State University, City of Sterlitamak, \\ Bashkortostan, Russia
}

\section{Summary}

The concept of space-matter motion in the new Cartesian physics, based on the identity of space and matter, creates the basis for the study of consciousness as the action of the brain in space inside and outside itself and offers a way of materialistic explanation of life on Earth. She claims that consciousness in living matter arises when the brain begins to create the surrounding space the image of themselves and the world. And since space according to Descartes is identical to matter, the images created by the brain of itself and the external world in the surrounding space have a material basis and therefore the displayed organs interact with each other and the external world.

Focusing on the identity of space and matter, New Cartesian Physics, which is a modern version of Descartes 'physics, provides a complete specification of space as moving matter and argues that the space-matter inside things is not limited to their visible boundary, but passes through them to the state of physical vacuum forming the Universe. Thus, it creates the basis for the study of intelligence as the work of the brain adjacent space inside and outside itself. Such a study was previously hampered by the conviction that a person exists in a void and all thought processes take place inside his head. The discovery of a material property in the surrounding space, which makes it identical to things, makes it possible to see the absence of the supernatural in the existence of living matter.

Its attribute, abstracted from matter, length, which was called space, is remembered by man as a possibility of movement among bodies and therefore it is perceived as existing separately from bodily matter [1]. In addition, mathematics invented a method for describing space as existing separately from the bodies. Thus, the unity of the objective world was divided into two categories existing separately from each other - body matter and space, as an empty repository of bodies.

Our ordinary consciousness is allowed to think that we exist in empty space, because it needs a sense of freedom, without which it cannot move. However, our scientific consciousness needs the conviction that space is matter, more precisely, an attribute of matter in order to overcome a crisis, exhausting the scientific community and diverting significant material resources to test erroneous hypotheses, because of its unwillingness to admit that space is it is matter and matter is space. The truth lies on the surface, and the scientific community walks around and stubbornly does not want to recognize it. This situation is similar to the denial of the Copernican heliocentric system, in which humanity for a long time did not want to believe.

It is necessary to convince all scientists that space is matter and that it is in motion. After all, when Copernicus began to assert that the earth revolves around the sun, he did not add that the whole solar space rotates with the earth. The conviction of the 
correctness of the Copernican heliocentric system indicates that a person is educated, and the recognition of the rotation of the near-solar space should be a sign of his intellect [2].

Only after New Cartesian Physics appears as a whole, can we begin to discuss it and make new discoveries based on it. At this moment, its significance can be judged by the fact that it has already explained the formula of mass-energy equivalence, and also found a connection between the Lorentz factor and the probability density of electron states [3]. Further generalization of physics and physiology based on the identity of space and matter will give us an understanding of the functioning of the intellect in the physical world.

Consciousness in the brain turns on at the moment when it begins to create in the space around itself an image of the external world according to signals coming from it. Since space is a moving matter, it is characterized by inertia, as a result of which the images created by the brain can exist for some time without it, creating paranormal and supernatural phenomena that are not recognized by official science. However, evidence of the existence of these phenomena is, both in the past and in the present and, obviously, will be in the future. The question arises - why does modern physics avoid them? What prevents her from recognizing their existence?

To answer this question, a critical approach to the scientific picture of nature, which creates and studies physics, is necessary. In general, this pattern consists of empty space and bodies moving in it. Further in the empty space there is a physical vacuum and a moving electromagnetic wave. All bodies have a surface that separates them from empty space and forms the boundary beyond which another body cannot penetrate without violent resistance. Everything inside the body is held by the forces of attraction [4]. In the empty space, bodies move from the first blow of God and are held in their orbits due to the forces of gravity, etc.

Our minds are so used to this picture that they say that this is absolutely true. Everything that happens outside of this picture, science declared paranormal and supernatural.

New Cartesian physics, based on the equivalence of space and matter, looks at the real world in a different way. For her, the whole world is a space that moves relative to him and all that follows from this, all of his condition. Particles, planets, stellar and galactic systems are the vortex state spaces. In New Cartesian Physics, a physical vacuum is a state of space in which there are no vortices, if they arise, but only for a short time. Electromagnetic waves are the oscillations of space. There are no clear boundaries between objects resulting from the rotation of space, for example, corpuscles [5]. The space between fixed objects participates in the creation of nonstationary paranormal and supernatural phenomena, since it is also material, like the objects themselves.

In New Cartesian Physics, the relocation of regions of space-matter occurs due to the absence of "bricks" in nature, from which absolutely dense spaces could be combined. In any case, there will be virtual emptiness between areas of space-matter. In an effort to fill the existing void of cosmic matter, its regions are in constant and infinite rotational motion, since space is an environment in which only closed motion is possible. Like the one who literally said: "movement is a synonym for emptiness". Filling cavities with the maximum possible speed, that is, at the speed of light, cosmic matter becomes homogeneous, practically without them, since their detection requires a speed greater than the speed of light, which is absent in nature. In fact, it turns out that they are no longer emptiness in the usual sense, but, together with moving regions of quanta, represent the motion of space-matter. These movements in space-matter in the state of physical vacuum are not given to man in contemplation, just as he was 
not given the opportunity to see the path of electromagnetic waves, which are also his oscillations. Particles, planets, stellar and galactic systems are pointers that indicate the movement of space. In the infinitely small regions of space, it rotates at the speed of light and angular momentum equal to the Planck constant, creating pressure [6]. Separate areas of space move in such a way that this pressure in the entire Universe is constant. The bodies move when this constancy is disturbed around them, as a result of any impact on them. The brain, creating images of action in space, structures it in such a way that the body moves in the required direction.

\section{References}

1. Dizhechko BS. LAMBERT Academic Publishing: Moving space-matter "(Part 1), 2012-10-01.

2. Ref.: https://goo.gl/h6fsYZ

3. Dizhechko BS. Behavior of vacuum holes in moving space-matter. Ref.: https://goo.gl/U6MWR6

4. Dizhechko BS. Principle of physical irrationality. Ref.: https://goo.gl/n8npkg

5. Dizhechko BS. The Law of Constancy of a Stream of Forces. Ref.: https://goo.gl/uLFavi

6. Dizhechko BS. Universe pressure. Ref.: https://goo.gl/B86FGX 